\title{
PROBABILIDADE DE OCORRÊNCIA DE CHUVAS EXTREMAS PARA REGIÃo DE INCONFIDENTES - MG
}

\author{
CAETANO, Alessander Lopes - alessander.lc@hotmail.com \\ Instituto Federal do Sul de Minas / IF Sul de Minas - Campus Inconfidentes \\ BARBOSA, Fernando da Silva - fernando.barbosa@ifsuldeminas.edu.br \\ Instituto Federal do Sul de Minas / IF Sul de Minas - Campus Inconfidentes
}

\begin{abstract}
RESUMO: O planejamento e desenvolvimento de urbanização, bem como de áreas para a agricultura, devem considerar as estimativas probabilísticas de chuvas fundamentais, para prever eventos raros ou extremos, que possuem grandes relevâncias climatológicas, hidrológicas e sociais. $O$ presente trabalho teve como objetivo estudar a probabilidade de ocorrência de chuvas e com esses dados saber se a precipitação segue um padrão ao longo dos anos e se sofre grandes variações (anomalias). O estudo foi realizado no IFSULDEMINAS-Campus Inconfidentes, utilizando os dados coletados na estação pluviométrica instalada nas margens do rio Mogi-Guaçu e que é mantida pela Agência Nacional de Águas (ANA) e operada pela Companhia de Pesquisa de Recursos Minerais (CPRM). Os dados obtidos correspondem aos anos de 1967 a 2015 e foi adotado um método simples de calcular probabilidade de ocorrência de chuva a partir de uma sequência de valores medidos, baseando-se na ordenação decrescente dos valores. A média anual de precipitação para Inconfidentes-MG foi de $1471,6 \mathrm{~mm}$ e analisando a série histórica não se observa tendências de aumento ou redução nas precipitações médias mensais, nem modificação no perfil de distribuição das chuvas ao longo dos anos estudados. Verificou-se que a probabilidade de ocorrência de chuvas extremas para Inconfidentes-MG é variável para cada mês, sendo os eventos mais críticos ocorridos nos meses de janeiro, fevereiro e outubro, com a probabilidade de 4,4\%, 2,2\% e 6,7\%, respectivamente, de ocorrer chuvas com mais de $90 \mathrm{~mm}$ diários e menor incidência nos meses de junho e julho com probabilidade de aproximadamente $0 \%$. Sabendo a disponibilidade hídrica, a probabilidade de ocorrência de eventos extremos e o período de retorno pode-se fazer um planejamento otimizado, em diversas áreas desde projetos ambientais a projetos de construções civis.
\end{abstract}

Palavras-chave: Precipitação; Anomalia Climática; Enchentes; Período de Retorno; Meio Ambiente.

\section{PROBABILITY OF EXTREME RAIN RAINS FOR THE INCONFIDENT REGION - MG}

ABSTRACT: The planning and development of urbanization, as well as areas for agriculture, should consider the probabilistic estimates of rainfall, to predict rare or extreme events, which have great climatological, hydrological and social relevance. The present study aimed to study the probability of occurrence of rainfall and with these data to know if the precipitation follows a pattern over the years and if it suffers great variations (anomalies). The study was carried out at the IFSULDEMINAS-Campus Inconfidentes, using the data collected at the pluviometric station installed on the banks of the Mogi-Guaçu River and maintained by the National Water Agency (ANA) and operated by the Mineral Resources Research Company (CPRM). The data obtained correspond to the years 1967 to 2015 and a simple method of calculating probability of occurrence of rain from a sequence of measured values was adopted, based on the descending ordering of the values. The mean annual rainfall for Inconfidentes-MG was $1471.6 \mathrm{~mm}$ and, analyzing the historical series, there were no trends of increase or reduction in average monthly rainfall, nor a change in rainfall distribution profile over the years studied. It was verified that the probability of occurrence of extreme rainfall for Inconfidentes-MG is variable for each month, with the most critical events occurring in January, February and October, with a probability of $4.4 \%, 2.2 \%$ and $6.7 \%$, respectively, of rainfall with more than $90 \mathrm{~mm}$ daily and lower incidence in the months of June and 
July with probability of approximately $0 \%$. Knowing the water availability, the probability of occurrence of extreme events and the period of return can be optimized planning, in several areas from environmental projects to civil construction projects.

Keywords: Precipitation; Climate Anomaly; Floods; Return Period; Environment. PROBABILITE DES PLUIES DE PLUIE EXTREME POUR LA REGION INCONFIDENTE - MG

RÈSUMÈ: La planification et le développement de l'urbanisation, ainsi que les domaines de l'agriculture, devraient tenir compte des estimations de probabilité des pluies fondamentales pour prédire des événements rares ou extrêmes qui ont une grande pertinence climatologique, hydrologique et social. Cette étude visait à étudier la probabilité de précipitations et avec ces données si la précipitation suit un modèle au cours des années et si vous avez de grandes variations (anomalies). L'étude a été menée dans Inconfidentes IFSULDEMINAS-Campus en utilisant les données recueillies dans la station pluviometric installée sur les rives de la rivière Mogi-Guaçu et qui est maintenue par l'Agence nationale de l'eau (ANA) et exploité par la Société de recherche des ressources minérales (CPRM). Les données correspondent à 1967-2015 et a été adopté une méthode simple de calcul de la probabilité d'occurrence de pluie à partir d'une séquence de valeurs mesurées, sur la base de l'ordre décroissant de valeurs. La pluviométrie moyenne annuelle pour Inconfidentes-MG était $1471,6 \mathrm{~mm}$ et d'analyse des séries historiques n'a pas observé une tendance croissante ou la réduction de la pluviométrie moyenne mensuelle, ou changement dans le mode de répartition des précipitations au cours des années étudiées. Il a été constaté que la probabilité de précipitations extrêmes pour Inconfidentes-MG est variable pour chaque mois, et les événements les plus critiques dans les mois de Janvier, Février et Octobre, avec la probabilité de $4,4 \%, 2,2 \%$ et $6,7 \%$ respectivement des précipitations avec plus de 90 $\mathrm{mm}$ par jour et une incidence plus faible en juin et juillet avec une probabilité d'environ $0 \%$. La connaissance de la disponibilité de l'eau, la probabilité d'occurrence d'événements extrêmes et la période de récupération peut faire une planification optimisée dans plusieurs domaines de projets environnementaux à des projets de travaux publics.

Mots Clés: précipitations; Anomalie climatique; Inondations Période de retour; Environnement

\section{PROBABILIDAD DE LLUVIAS EXTREMAS PARA LA REGIÓN INCONFIDENTE - MG}

RESUMEN: La planificación y el desarrollo de la urbanización, así como de áreas para la agricultura, deben considerar las estimaciones probabilísticas de lluvias fundamentales, para predecir eventos raros o extremos, que tienen grandes relevancia climatológica, hidrológica y social. El presente trabajo tuvo como objetivo estudiar la probabilidad de ocurrencia de lluvias y con esos datos saber si la precipitación sigue un patrón a lo largo de los años y si sufre grandes variaciones (anomalías). El estudio fue realizado en el IFSULDEMINAS-Campus Inconfidentes, utilizando los datos recolectados en la estación pluviométrica instalada en las márgenes del río Mogi-Guaçu y que es mantenida por la Agencia Nacional de Aguas (ANA) y operada por la Compañía de Investigación de Recursos Minerales (CPRM). Los datos obtenidos corresponden a los años de 1967 a 2015 y se adoptó un método simple de calcular la probabilidad de ocurrencia de lluvia a partir de una secuencia de valores medidos, basándose en la ordenación decreciente de los valores. La media anual de precipitación para Inconfidentes-MG fue de 1471,6 mm y analizando la serie histórica no se observan tendencias de aumento o reducción en las precipitaciones medias mensuales, ni modificación en el perfil de distribución de las lluvias a lo largo de los años estudiados. Se verificó que la probabilidad de ocurrencia de lluvias extremas para Inconfidentes-MG es variable para cada mes, siendo los eventos más críticos ocurridos en los meses de enero, febrero y octubre, con la probabilidad del $4,4 \%$, el $2,2 \%$ y el $2,2 \%$ El $6,7 \%$, respectivamente, de ocurrir lluvias con más de $90 \mathrm{~mm}$ diarios y menor incidencia en los meses de junio y julio con probabilidad de aproximadamente $0 \%$. Sabiendo la disponibilidad hídrica, la probabilidad de ocurrencia de eventos extremos y el período de retorno se puede hacer una planificación 
optimizada, en diversas áreas desde proyectos ambientales a proyectos de construcciones civiles.

Palabras Clave: Precipitación; Anomalía Climática; inundaciones; Período de Retorno; Medio ambiente.

\section{INTRODUÇÃO}

Em função das exigências térmicas e hídricas de um determinado cultivo (consumo ou reflorestamento), uma região pode ser classificada como apta, marginal, ou inapta para implantação de uma cultura, sendo assim, o conhecimento da disponibilidade hídrica regional é uma das bases para elaboração do Zoneamento Agroclimático de uma cultura, podendo indicar as melhores épocas de semeadura para que as plantas estejam menos sujeitas a restrições hídricas durante seu crescimento, alcançando máxima produção com uso consciente dos recursos naturais disponíveis para aquela região.

Porém, não só o conhecimento da distribuição das chuvas, refletido no Balanço Hídrico, é importante para um manejo conservacionista do solo e da água (SANTOS; GRIEBELER; OLIVEIRA, 2009). É necessário, também, o conhecimento da ocorrência de eventos extremos, como precipitações máximas diárias e precipitação total mensal, que não se destacam no Balanço Hídrico Climatológico, mas que causam grandes problemas/tragédias tanto em áreas agrícolas como urbanas. Neste contexto, podem ser destacadas as enchentes ocorridas no início de 2016, em Inconfidentes-MG, que chegaram a desabrigar mais de 300 famílias.

Para a elaboração de um trabalho que apresente informações climáticas concretas e com precisão, há a dificuldade de analisar, organizar e corrigir, se necessários, todos os dados de no mínimo de um período de 30 anos e com esses dados, transformar as informações de utilidade para várias áreas do conhecimento.

Assim, o estudo da Probabilidade de ocorrência de chuvas extremas apresenta importância para a região em estudo, tendo em vista não só aptidão agrícola da região, mas os problemas de caráter urbano, como sistemas de drenagem, que podem ser mais bem projetados/dimensionados com base neste estudo.

Este trabalho teve como objetivo estudar a probabilidade de ocorrência de chuvas extremas utilizando dados históricos de precipitações locais para região de Inconfidentes - MG disponibilizados pela Agência Nacional de Águas (ANA), e com esses dados verificar se a precipitação segue um padrão ao longo dos anos e se sofre grandes variações.

\section{A VARIABILIDADE CLIMÁTICA}

A Variabilidade Climática pode ser entendida como uma característica natural do sistema climático terrestre, responsável por oscilações naturais nos padrões climáticos, notados em nível local, regional e global (CONFALONIERI, 2003). No caso do Brasil, a disponibilidade de água depende em grande parte do clima, sendo que o ciclo anual das chuvas e de vazões nas bacias hidrográficas variam, e de fato, a variabilidade interanual do clima, associada aos fenômenos climáticos, como exemplo o El Niño ou a La Niña, podem gerar 
anomalias climáticas, que produzem grandes secas ou chuvas intensas (MARENGO, 2008).

As anomalias climáticas são eventos meteorológicos e climatológicos que fogem dos valores médios, como exemplo a precipitação, que pode ser muito superior ao valor médio ou muito abaixo, gerando uma anomalia que pode causar desde enchentes até grandes períodos de estiagem (PEREIRA; ANGELOCCI; SENTELHAS, 2007).

A precipitação pluvial, ou chuva, é o principal componente do ciclo hidrológico, pois representa a maior entrada de água na bacia hidrográfica. Quando ocorre o aumento dessa precipitação há uma situação crítica, denominada de eventos extremos ou chuvas intensas. Sendo que, um evento pluviométrico intenso é considerado para valores superiores ao percentil de $95,0 \%$ de uma série de dados, enquanto, o evento pluviométrico extremo, é considerado para valores superiores ao percentil de 99,0\% da mesma série de dados. A análise dos impactos gerados pelas Chuvas Extremas é de total importância para sociedade, pois determina áreas de riscos para habitação e agricultura (MELLO; VIOLA, 2013). Também deve-se analisar as chuvas intensas para o planejamento de obras hidráulicas de controle de cheias, drenagem urbana e do solo e abastecimento urbano e/ou rural (MELLO; VIOLA; MELLO, 2007).

\section{PROBABILIDADE DE OCORRÊNCIA}

A estimativa da probabilidade de ocorrência de Chuvas Extremas, como o caso da Precipitação Diária Máxima Anual (PDMA), tem uma forte motivação para seu estudo, pois podem ser potencialmente nocivos à sociedade (JUNQUEIRA JÚNIOR; MELLO; ALVES, 2015).

Para se ter um ótimo planejamento e desenvolvimento de urbanização, assim como de áreas para a agricultura, as estimativas probabilísticas são fundamentais, sendo usadas para estimar eventos raros ou extremos, que possuem grande relevância climatológicas, hidrológicas e sociais (SANSIGOLO, 2008).

Eventos Climáticos Extremos ocorrem de muitas maneiras, como enchentes, secas prolongadas, tufões, tornados e ondas de calor. Estes fenômenos naturais não são novidades, pois através de séculos são observados pelo homem, que desenvolveu uma boa percepção da frequência e de ordem em que mais ocorrem no nosso planeta. Mas isto está mudando cada vez mais e a partir da segunda metade do século XX, observou que devido à ação antrópica estes eventos vêm se intensificando e acontecendo com maiores frequências (MARENGO, 2009).

O excesso de precipitação muitas vezes causa alagamentos e deslizamentos de terra e a ausência pode ocasionar longos períodos de seca prejudicando a agricultura e o abastecimento populacional (ALVARENGA, 2012).

Os efeitos devastadores sobre a população que habita zonas urbanas, causados pelas enchentes ocorrem devido ao pouco planejamento e uso incorreto das áreas de ocupação (BRITO; FEITOSA; NASCIMENTO, 2015), que poderiam ser evitados ou corrigidos com o conhecimento do histórico destes eventos para o correto planejamento urbano. A estimativa da probabilidade de ocorrência de precipitações diárias máximas anuais pode ser local, ou seja, 
utilizando-se a série de dados de uma única estação, ou regional, fazendo uso dos dados de várias estações de uma região (WESCHENFELDER; PICKBRENNER; PINTO, 2011).

\section{MATERIAL E MÉTODO}

O trabalho foi realizado no IFSULDEMINAS - Campus Inconfidentes, localizado no município de Inconfidentes - MG, Brasil. O clima da região, segundo a classificação de Koëppen é do tipo subtropical de inverno seco e verão quente (Cwa), com duas estações definidas: chuvosa (outubro a março) e seca (abril a setembro), com médias anuais de precipitação e temperatura de $1.800 \mathrm{~mm}$ e $19^{\circ} \mathrm{C}$, respectivamente (PEREIRA; BALLEIRO; PINTO, 2010).

Foram analisados no trabalho os dados coletados na estação pluviométrica instalada no município de Inconfidentes-MG nas margens do rio Mogi-Guaçu. Esta estação é mantida pela Agência Nacional de Águas (ANA) e operada pela Companhia de Pesquisa de Recursos Minerais (CPRM). Os dados utilizados estão disponíveis no Hidroweb (http://www.snirh.gov.br/hidroweb/) e a estação localizada nas coordenadas geográficas com latitude $22^{\circ} 19^{\prime} 07.1^{\prime \prime S}$ e longitude 46¹9'17.9"W (Figura 1).

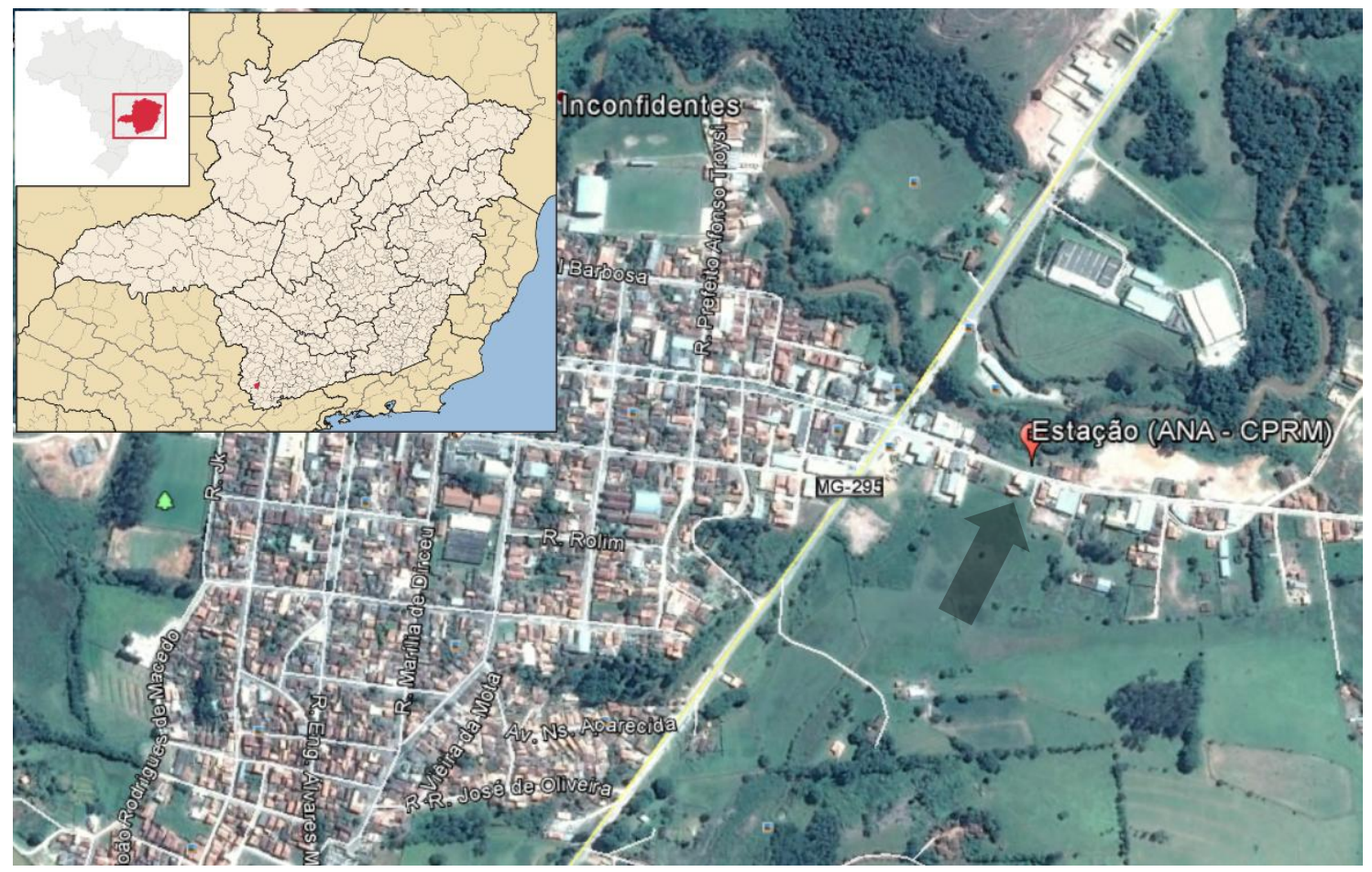

Figura 1 - Localização da estação pluviométrica.. Fonte: Google Earth Pro; Wkipedia. 


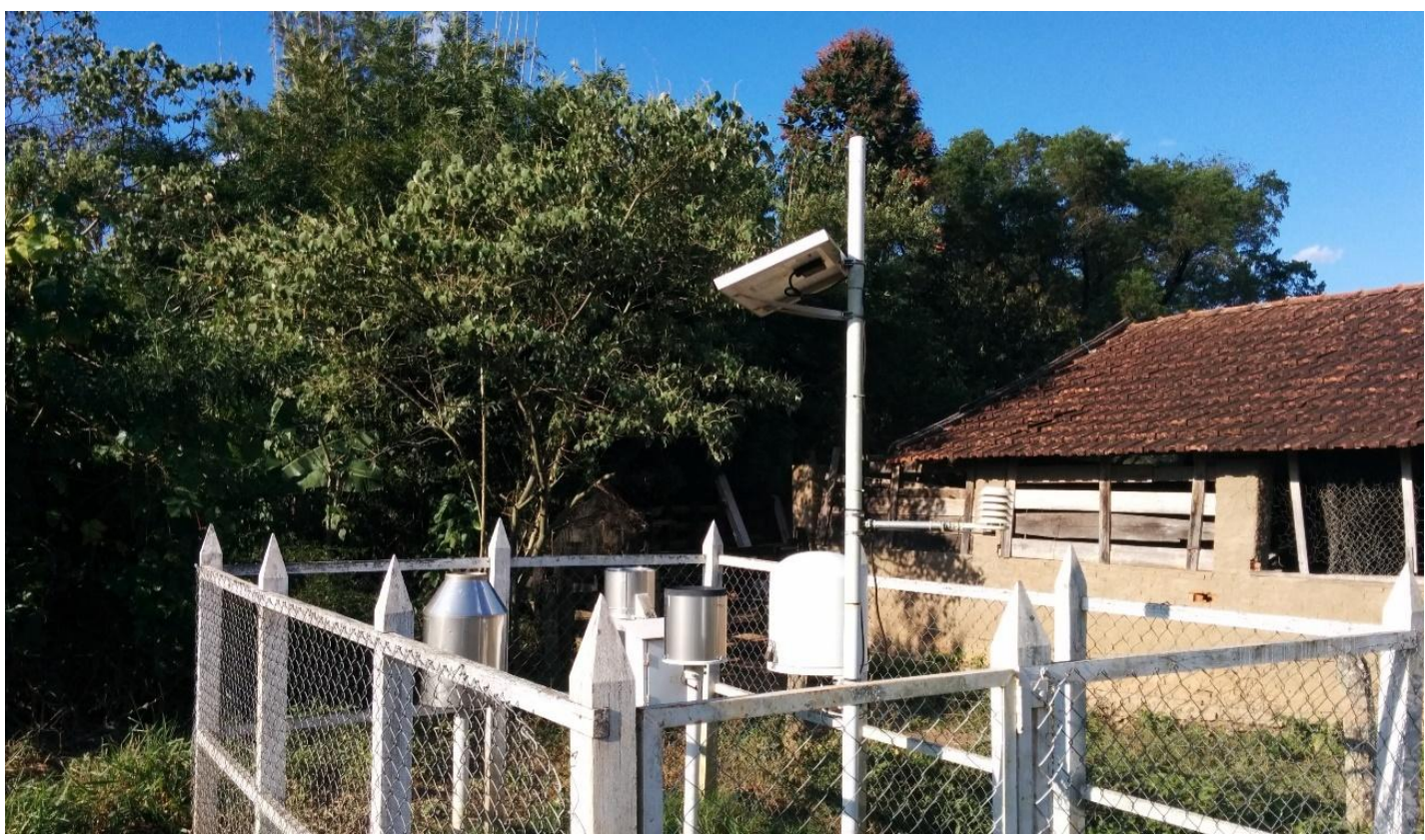

Figura 2 - Vista geral da estação pluviométrica. Fonte: Acervo do autor.

Os dados obtidos foram de 1967 a 2015, sendo desconsiderados os anos de 1972, 1991, 1992, 2006 e 2007 por falta de consistência; os 44 anos restantes foram analisados e deixados em conformidades (preenchimento de falhas e ordenação), proporcionando uma série histórica consistente. Com esses dados foi possível obter informações como, máxima diária, média de precipitação mensal e anual e mínima/máxima mensal; que possibilitou a geração dos gráficos.

Com a intenção de observar se a precipitação segue um padrão nos últimos 48 anos houve a divisão de 4 períodos de 12 anos cada. (Figura-04)

Foi adotado um método simples de calcular a probabilidade de ocorrência de chuvas a partir de uma sequência de valores medidos, baseando-se na ordenação crescente ou decrescente dos valores. Se a ordenação for crescente, a probabilidade corresponderá a um valor igual ou menor que o limite escolhido; se for decrescente, estima-se o inverso. (PEREIRA; ANGELOCCI; SENTELHAS, 2007).

Portanto, para saber a probabilidade de ocorrência de chuvas $(P)$ de um valor maior ou menor que um valor crítico, foi ordenada a sequência de dados em ordem decrescente, respectivamente, e divide-se o número de ordem (m) correspondente à posição do valor crítico pelo número total de dados (n) mais 1 , como destacado na equação 1 :

$$
\mathrm{P}=\frac{\mathrm{m}}{\mathrm{n}+1} * 100
$$

Onde:

P:Probabilidade de ocorrência em \% 
m: Número da ordem

$\mathrm{n}$ :Número de dados total

Se na sequência de dados houver ocorrência de valores nulos (ausência de chuva), eles são descartados, anotando-se quantas vezes isto ocorreu (No), fazendo-se depois a ordenação dos valores restantes. Nesse caso, a probabilidade de ocorrência $(P)$ de um valor crítico será dada pela seguinte relação:

$$
P=\left(1-\frac{\text { No }}{n}\right)\left(\frac{m}{n+1-N o}\right) * 100
$$

Equação: (2)

Onde:

P:Probabilidade de ocorrência em \%

No: Número de valores nulos de precipitação

$\mathrm{n}$ : Número de dados total

$\mathrm{m}:$ Número da ordem

Uma informação importante quando se trabalha com probabilidade de ocorrência de um elemento meteorológico é o período de retorno ou intervalo médio de recorrência, que é representado pelo símbolo t. Interpreta-se período de retorno como sendo o tempo provável esperado que aquele fenômeno ocorra novamente. Geralmente, calcula-se o período de retorno para valores extremos, ou seja, nos casos de anomalias climáticas que podem causar algum impacto econômico e social, e não para aqueles valores que ocorrem normalmente. Para que a estimativa de t seja a melhor possível, é necessário que se disponha de uma série longa de valores medidos, pois $t$ depende da probabilidade de ocorrência $(P)$ do fenômeno. retorno.

Quanto mais frequente o valor crítico analisado, menor será o período de

Ordenação decrescente:

$$
\mathbf{t}=\mathbf{1} / \mathbf{P} \text {. }
$$

Equação:(3)

Onde:

t: Período de retorno, em anos;

P: Probabilidade de ocorrência, em decimal.

Com os valores de probabilidade e período de retorno foram adotadas as precipitações de $50 \mathrm{~mm}, 60 \mathrm{~mm}, 70 \mathrm{~mm}, 80 \mathrm{~mm}$ e $90 \mathrm{~mm}$ para valores diários e $25 \mathrm{~mm}, 50 \mathrm{~mm}, 150 \mathrm{~mm}, 300 \mathrm{~mm}$ e $500 \mathrm{~mm}$ para o total mensal para análise seguindo as equações 1 e/ou 2 e 3, com base nos dados históricos analisados.

Em seguida foram elaboradas 4 tabelas com os dados obtidos, apresentando as probabilidades e períodos de retornos. As tabelas apresentam 
os valores em porcentagem de probabilidade de ocorrência máxima diária; o tempo de período de retorno diário em anos; os valores em porcentagem de probabilidade de ocorrência total mensal; o período de retorno total mensal em anos.

\section{RESULTADOS E DISCUSSÃO}

Os resultados obtidos, em primeiro plano, foram as médias, as mínimas e as máximas mensais das chuvas no período de 1967-2015.

A média anual de precipitação para Inconfidentes-MG foi de $1471,6 \mathrm{~mm}$, que não se distanciou dos $1500,0 \mathrm{~mm}$ anuais apresentados no balanço hídrico disponível no site CLIMATE-Data.ORG para a cidade de Inconfidentes-MG.

Analisando as médias mensais de chuvas observou-se que InconfidentesMG possui meses como dezembro e janeiro com elevados valores de precipitação, chegando a mais de $500 \mathrm{~mm}$ e meses como junho e julho onde há redução de chuvas, chegando até a $0 \mathrm{~mm}$ de precipitação mensal. (Figura-03)

A precipitação mínima mostrou que mesmo em anos de déficit de chuvas, janeiro e dezembro chovem quantidades significativas, mas nos meses como abril, junho, julho e agosto podem chegar à zero de precipitação mesmo em anos mais chuvosos.

Por outro lado, houve anos em que dezembro chegou a ter altíssimas precipitações com mais de $500 \mathrm{~mm}$ (1986) no total mensal e Janeiros com mais de $466 \mathrm{~mm}$ (1989) no total mensal.

A média mensal do mês de dezembro foi de $241,9 \mathrm{~mm}$, sendo observado que em dezembro de 1986 choveu um total de $509 \mathrm{~mm}$, considerado uma anomalia climática, pois choveu mais que o dobro da média para esse período.

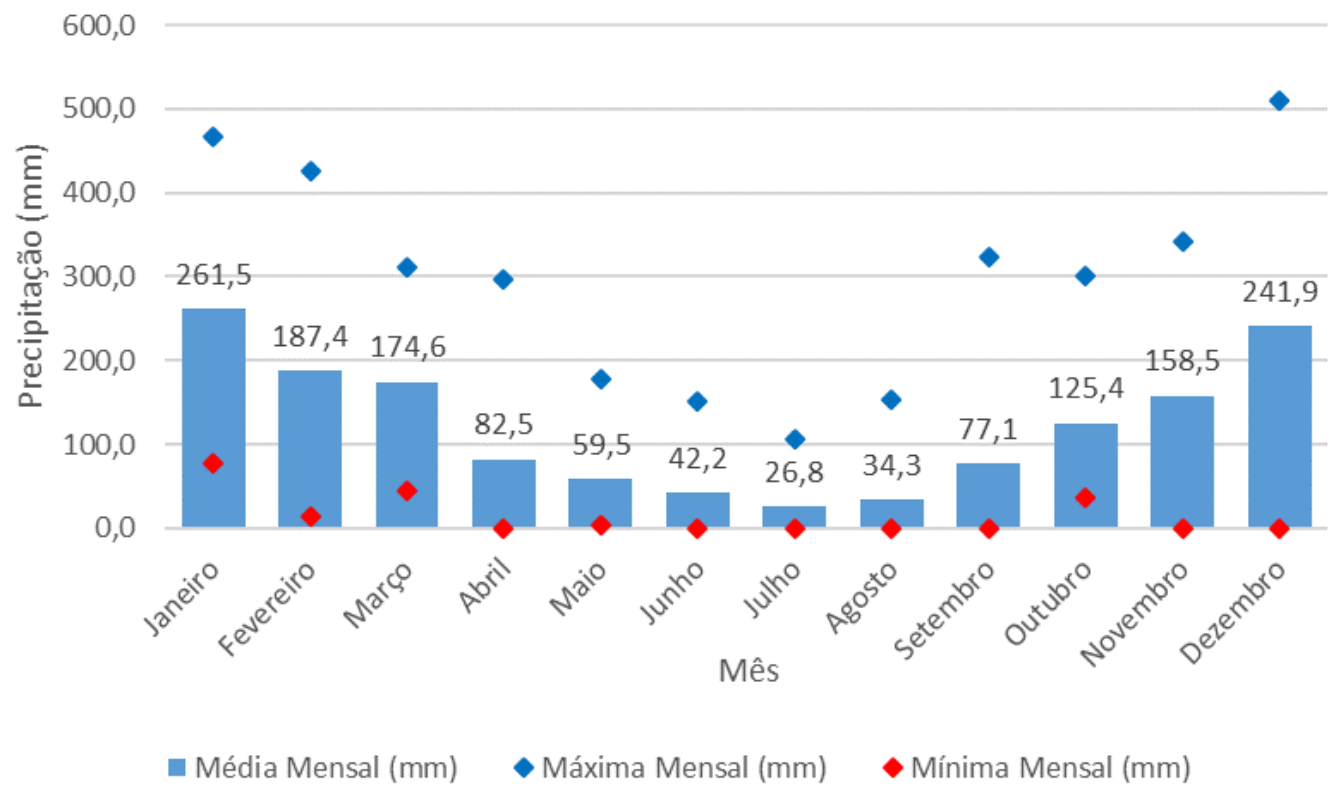


Figura 3 - Distribuição média, mínima e máxima mensal das chuvas no período de 1967 - 2015, para Inconfidentes, MG.

Com a divisão de 4 períodos de 12 anos cada, dos 48 anos estudados notou-se que a precipitação segue um padrão no qual tem uma notável presença de janeiros e dezembros chuvosos, e junhos e julhos secos; definindo verões úmidos e invernos secos (Figura 4).

Analisando-se os 4 períodos comparativamente não observa-se tendências de aumento ou redução nas precipitações médias mensais, nem modificação no perfil de distribuição das chuvas ao longo dos anos estudados (Figura 4).

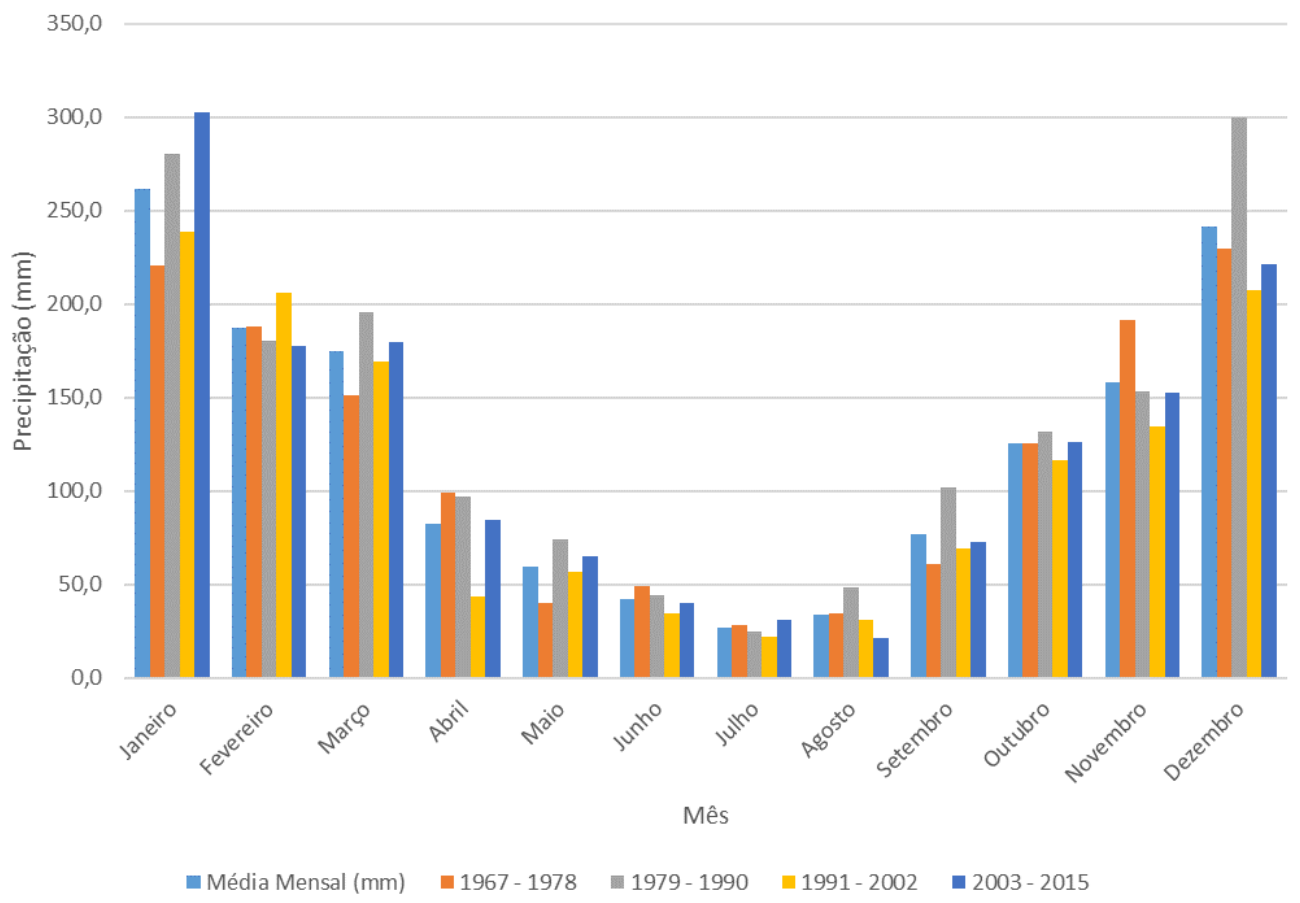

Figura 4 - Distribuição média das chuvas mensais no período de 1967 - 2015, dividido em quatro períodos de doze anos para o município de Inconfidentes, MG.

Em relação a análise de probabilidade de ocorrência pode-se observar na Tabela 01 que há a chance de no mês de janeiro, fevereiro e outubro ocorrer chuvas diárias que ultrapassam $90 \mathrm{~mm}$ diários. Para os demais meses, eventos dessa magnitude não foram observados no período estudado.

Observando a Tabela 01 , verifica-se que todos os meses há uma probabilidade mínima de ocorrer chuvas de até $50 \mathrm{~mm}$ diários, com exceção do mês de julho, isto porque no período avaliado, em nenhum mês de julho ocorreu uma precipitação acima de $50 \mathrm{~mm}$.

Destaca-se que a chuva que causou a enchente em janeiro 2016 em Inconfidentes-MG, desabrigando mais de 300 famílias foi de aproximadamente $110 \mathrm{~mm}$ diário, segundo os dados coletados pela estação meteorológica do IFSULDEMINAS - Campus Inconfidentes, sendo o maior valor registrado para o período disponível de dados da instituição. E analisando a série de dados estudados neste trabalho também não havia sido registrado nem um valor tão alto de precipitação diária para o mês de janeiro. 
Tabela 01 - Probabilidade de Ocorrência (\%) de precipitação máxima diária acima dos valores críticos estabelecidos.

\begin{tabular}{ccccccccccccc}
\hline Precipitação & \multicolumn{110}{c}{ Mêses } \\
\cline { 2 - 12 } Crítica (mm) & JAN & FEV & MAR & ABR & MAI & JUN & JUL & AGO & SET & OUT & NOV & DEZ \\
\hline 50,0 & 42,2 & 26,7 & 22,2 & 11,1 & 4,4 & 6,7 & $* 0$ & 2,2 & 6,7 & 8,9 & 2,2 & 33,3 \\
60,0 & 24,4 & 11,1 & 8,9 & 4,4 & 2,2 & $* 0$ & $* 0$ & 2,2 & 2,2 & 8,9 & 13,3 & 17,8 \\
70,0 & 15,6 & 6,7 & 6,7 & 2,2 & 2,2 & $* 0$ & $* 0$ & $* 0$ & 2,2 & 4,4 & $* 0$ & 8,9 \\
80,0 & 6,7 & 4,4 & 2,2 & $* 0$ & 2,2 & $* 0$ & $* 0$ & $* 0$ & $* 0$ & 2,2 & $* 0$ & 6,7 \\
90,0 & 4,4 & 2,2 & $* 0$ & $* 0$ & $* 0$ & $* 0$ & $* 0$ & $* 0$ & $* 0$ & 2,2 & $* 0$ & $* 0$ \\
\hline
\end{tabular}

* Dentro do período analisado não houve precipitação desta magnitude.

Observa-se na Tabela 02 os dados de período de retorno, indicando chuvas de alta intensidade diária, consideradas críticas, que ocorrem em um período mínimo de 22 anos para o mês de janeiro e 45 anos para o mês de fevereiro e outubro, considerando uma chuva acima de $90 \mathrm{~mm}$ diária. Considerando este mesmo valor de precipitação os demais meses terão um período de retorno acima de 45 anos.

Já para a precipitação acima de $50 \mathrm{~mm}$ diária os meses de janeiro e dezembro possuem período de retorno abaixo de 3 anos, demostrando que essa intensidade de precipitação é muito frequente nestes meses, dando um indicativo da importância da atividades de conservação do solo nestes períodos.

Tabela 02 - Período de retorno (anos) para precipitação máxima diária acima dos valores críticos estabelecidos.

\begin{tabular}{ccccccccccccc}
\hline Precipitação & \multicolumn{10}{c}{ Mêses } \\
\cline { 2 - 12 } Crítica (mm) & JAN & FEV & MAR & ABR & MAI & JUN & JUL & AGO & SET & OUT & NOV & DEZ \\
\hline 50,0 & 2,4 & 3,8 & 4,5 & 9,0 & 22,5 & 15,0 & $*>45$ & 45,0 & 15,0 & 11,3 & 4,5 & 3,0 \\
60,0 & 4,1 & 9,0 & 11,3 & 22,5 & 45,0 & $*>45$ & $*>45$ & 45,0 & 45,0 & 11,3 & 7,5 & 5,6 \\
70,0 & 6,4 & 15,0 & 15,0 & 45,0 & 45,0 & $*>45$ & $*>45$ & $*>45$ & 45,0 & 22,5 & $*>45$ & 11,3 \\
80,0 & 15,0 & 22,5 & 45,0 & $*>45$ & 45,0 & $*>45$ & $*>45$ & $*>45$ & $*>45$ & 45,0 & $*>45$ & 15,0 \\
90,0 & 22,5 & 45,0 & $*>45$ & $*>45$ & $*>45$ & $*>45$ & $*>45$ & $*>45$ & $*>45$ & 45,0 & $*>45$ & $*>45$ \\
\hline
\end{tabular}

* Dentro do período analisado não houve precipitação desta magnitude.

Logo para o total mensal (Tabela 3) observa-se que os meses de dezembro podem ocorrer chuvas com mais de $500 \mathrm{~mm}$ mensais e essa intensidade mensal como apresentado na Tabela 04 de período de retorno acontece no mínimo a cada 45 anos.

Tabela 03 - Probabilidade de Ocorrência (\%) de precipitação total mensal acima dos valores críticos estabelecidos.

\begin{tabular}{ccccccccccccc}
\hline Precipitação & \multicolumn{10}{c}{ Mêses } \\
\cline { 2 - 11 } Crítica (mm) & JAN & FEV & MAR & ABR & MAI & JUN & JUL & AGO & SET & OUT & NOV & DEZ \\
\hline 25,0 & 97,8 & 95,6 & 97,8 & 82,2 & 77,8 & 53,3 & 42,2 & 42,2 & 77,8 & 97,8 & 95,6 & 95,6 \\
50,0 & 97,8 & 93,3 & 95,6 & 68,9 & 48,9 & 35,6 & 20 & 28,9 & 62,2 & 86,7 & 95,6 & 95,6 \\
150,0 & 86,7 & 62,2 & 57,8 & 17,8 & 4,4 & 2,2 & $* 0$ & 2,2 & 6,7 & 33,3 & 55,6 & 80 \\
300,0 & 33,3 & 8,9 & 2,2 & $* 0$ & $* 0$ & $* 0$ & $* 0$ & $* 0$ & 2,2 & 2,2 & 4,4 & 28,9 \\
500,0 & $* 0$ & $* 0$ & $* 0$ & $* 0$ & $* 0$ & $* 0$ & $* 0$ & $* 0$ & $* 0$ & $* 0$ & $* 0$ & 2,2 \\
\hline
\end{tabular}

* Dentro do período analisado não houve precipitação desta magnitude. 
Tabela 04 - Período de retorno para precipitação total mensal acima dos valores críticos estabelecidos.

\begin{tabular}{ccccccccccccc}
\hline Precipitação & \multicolumn{10}{c}{ Mêses } \\
\cline { 2 - 14 } Crítica (mm) & JAN & FEV & MAR & ABR & MAI & JUN & JUL & AGO & SET & OUT & NOV & DEZ \\
\hline 25,0 & 1,0 & 1,0 & 1,0 & 1,2 & 1,3 & 1,9 & 2,4 & 2,4 & 1,3 & 1,0 & 1,0 & 1,0 \\
50,0 & 1,0 & 1,1 & 1,0 & 1,5 & 2,0 & 2,8 & 5,0 & 3,5 & 1,6 & 1,2 & 1,0 & 1,0 \\
150,0 & 1,2 & 1,6 & 1,7 & 5,6 & 22,5 & 45,0 & $*>45$ & 45,0 & 15,0 & 3,0 & 1,8 & 1,3 \\
300,0 & 3,0 & 11,3 & 45,0 & $*>45$ & $*>45$ & $*>45$ & $*>45$ & $*>45$ & 45,0 & 45,0 & 22,5 & 3,5 \\
500,0 & $*>45$ & $*>45$ & $*>45$ & $*>45$ & $*>45$ & $*>45$ & $*>45$ & $*>45$ & $*>45$ & $*>45$ & $*>45$ & $*>45$ \\
\hline
\end{tabular}

* Dentro do período analisado não houve precipitação desta magnitude.

As tabelas 03 e 04 acima apresentam informações que podem servir para saber quais meses que mais possuem chances de secas ou que podem ter alta taxa de precipitação e de quanto em quanto tempo podem ocorrer tais eventos novamente.

Determinando-se a disponibilidade hídrica, agricultores, engenheiros, responsáveis por gestão de cidades entre outros, podem fazer suas plantações, recuperações de matas ciliares/reflorestamento ou projetos baseados na probabilidade de ocorrência de chuvas, tendo noção de quanto, obras como barragens ou represas como exemplo, podem suportar, ou se para aquele ano haverá disponibilidade hídrica para determinada cultura agrícola, ou haverá chance de ocorrer uma enchente, podendo prevenir desastres.

De forma geral quase todos os meses, exceto no período de inverno, possuem disponibilidade hídrica de no mínimo $150 \mathrm{~mm}$ com um período de retorno inferior a 1 ou 2 anos, confirmando que esta precipitação tem uma grande frequência de ocorrência e pode ser considerada com um nível de segurança para atividades em geral.

\section{CONCLUSÕES}

A probabilidade de ocorrência de chuvas no município de Inconfidentes é variável para cada mês, mas segue um padrão no decorrer dos anos. Embora meses como janeiro, fevereiro e outubro, sejam mais críticos com a probabilidade de $4,4 \%, 2,2 \%$ e $6,7 \%$, respectivamente para ocorrência de chuvas com mais de $90 \mathrm{~mm}$ diários e menor incidência nos meses de junho e julho com probabilidade de aproximadamente $0 \%$.

A possibilidade de anomalias na precipitação são reais mesmo que com a baixa probabilidade.

Inconfidentes-MG possui alta disponibilidade hídrica, com maior volume de chuvas concentrado no período de verão, sendo uma região apta a várias culturas agrícolas.

Determinando a disponibilidade hídrica, a probabilidade de ocorrência de eventos extremos e o período de retorno pode-se fazer um planejamento otimizado, ajudando no desenvolvimento de vários tipos de projetos, trazendo benefícios para a sociedade local. 


\section{REFERÊNCIAS}

ALVARENGA, Lívia Alves. Precipitação no sudeste brasileiro e sua relação com a Zona de Convergência do Atlântico Sul. Revista Agrogeoambiental, Minas Gerais, v. 4, n. 2, p.01-07, ago. 2012.

BRITO, John Handerson do Nascimento; FEITOZA, Denise Magalhães Azevedo; NASCIMENTO, Nilson da Silva. Eventos extremos de precipitação no juazeiro do norte. In: 2 WORKSHOP INTERNACIONAL, 2., 2015, Município de Barbalha - CE. Resumo de Congresso. Município de Barbalha - CE: Centec, 2015. p. 1 - 8.

CONFALONIERI, Ulisses E. C.. Variabilidade climática, vulnerabilidade social e saúde no Brasil. Terra Livre, São Paulo, v. 1, n. 20, p.193-204, jul. 2003.

JUNQUEIRA JÚNIOR, José A.; MELLO, Carlos R. de; ALVES, Geovane J.. Eventos extremos de precipitação no Alto Rio Grande, MG: Análise probabilística. Revista Brasileira de Engenharia Agrícola e Ambiental, Campina Grande, v. 19, n. 4, p.301-308, mar. 2015.

MARENGO, José Antônio. Mudanças Climáticas e Eventos Extremos no Brasil. Rio de Janeiro: Fbds, 2009.

MARENGO, José Antônio. Água e mudanças climáticas. Estudos Avançados, São Paulo, v. 22, n. 63, p.83-96, 2008.

MELLO, Carlos Rogério de; VIOLA, Marcelo Ribeiro. Mapeamento de chuvas intensas no estado de Minas Gerais. Revista Brasileira de Ciência do Solo, Viçosa, v. 37, n. 1, p.37-44, fev. 2013.

MELLO, Carlos Rogério de; VIOLA, Marcelo Ribeiro; MELLO, José Marcio de. Continuidade espacial de chuvas intensas no Estado de Minas Gerais. Ciência e Agrotecnologia, Lavras, v. 32, n. 2, p.532-539, fev. 2007.

PEREIRA, A. R.; ANGELOCCI, L. R.; SENTELHAS, P. C. Meteorologia Agrícola. ESALQ, Departamento de Ciências Exatas. Piracicaba, SP. 2007. 192 p.

PEREIRA, Michender Werison Motta; BALIEIRO, Kátia Regina de Carvalho; PINTO, Lilian Vilela Andrade. Avaliação da produtividade e adaptabilidade de acessos de amendoim forrageiro para potencial formação/consorciação de pastagens mais sustentáveis no sul de Minas Gerais. In: II CONGRESSO BRASILEIRO DE GESTÃO AMBIENTAL, 2., 2011, Londrina- Paraná. Resumo de Congresso . Londrina: Ibeas, 2011. p. 1 - 6.

SANSIGOLO, Clóvis Angeli. Distribuições de extremos de precipitação diária, temperatura máxima e mínima e velocidade do vento em Piracicaba, SP (19172006). Revista Brasileira de Meteorologia, Piracicaba, v. 23, n. 3, p.341-346, mar. 2008.

SANTOS, Glenio G.; GRIEBELER, Nori P.; OLIVEIRA, Luiz F. C. de. Chuvas intensas relacionadas à erosão hídrica. Revista Brasileira de Engenharia Agrícola e Ambiental, Campina Grande, Paraíba, v. 14, n. 2, p.115-123, set. 2009.

WESCHENFELDER, Adriana Burin; PICKBRENNER, Karine; PINTO, Eber José de Andrade. Análise da frequência de ocorrência e a classificação das precipitações diárias máximas anuais na região da laguna dos patos (sub-bacia 87). In: XIX simpósio brasileiro de recursos hídricos. Resumo de Congresso. Maceió: Abril, 2011. p. $01-20$. 